\title{
Study of Biointegration and Tissue Reaction of Polyethylene Terephtalate 3D and Polypropylene Surgical Meshes in Rat Model
}

\author{
ZALAN BENEDEK ${ }^{2}$, ORSOLYA HANKO BAUER ${ }^{1,2 *}$, SORIN SORLEA ${ }^{1,2}$, MARIUS FLORIN COROS ${ }^{1,2}$, TIBOR MEZEI ${ }^{1,3}$, \\ RARES GEORGESCU1,2 \\ ${ }^{1}$ University of Medicine and Pharmacy Tîrgu Mures, Department of Surgery, 1 Gheorghe Marinescu Str., 540103, Targu Mures, \\ Romania \\ ${ }^{2}$ Surgical Clinic, Mures County Clinical Hospital, 1 Gheorghe Marinescu Str., 540103, Targu Mures, Romania \\ ${ }^{3}$ University of Medicine and Pharmacy Tirgu Mures, Department of Pathology, 50 Gheorghe Marinescu Str., 540136, Tirgu Mures, \\ Romania
}

\begin{abstract}
The aim of this experimental study is to compare the biointegration and tissue response to different surgical mesh types, inserted in various layers of the abdominal wall of Wistar rats. After 30 days, the rats were sacrificed and the meshes were removed en bloc with the subcutaneous tissue, muscular layer and peritoneum. Tissue response (fibrosis, inflammation) was measured using quantitative and semi-quantitative morphometry. We observed that polyethylene terephthalate 3D mesh induces significantly more fibrosis compared to polypropylene mesh and the fibrosis is absent or minimal in case of the control group. Tissue reaction, including fibrosis, is reduced around the polypropylene mesh. We also observed that fibrosis is more intense towards aponeurosis and peritoneum. We can conclude that the 3D mesh shows better biointegration if it is used in proper circumstances. It's well tolerated by the organism if sutured to the aponeurosis and preferable with monofilament stitches.
\end{abstract}

Keywords: abdominal wall hernia, surgical mesh, polyethylene terephtalate 3D, polypropylene

The abdominal wall reconstruction is a surgical intervention of large spectrum considering the patient, the characteristics of the defect and on the point of view of the surgical treatment, as well. This surgical intervention was revolutionized by exponentially increasing types of prosthetics and several innovative minimally invasive techniques the medicine is using nowadays. The reconstruction of the abdominal wall is a complex surgical treatment in development which ranges from a simple spotting of the muscle and stretches to the reconstruction of the whole abdominal wall. There does not exist a uniquely medical procedure or a surgical prosthesis which is applicable equally at any eventuality and which would be functional in any situation. That is the point the surgeon must decide independently at any separate case what procedure is the best customized for the patient [1-3].

A biomaterial is any matter, surface, or construct that interacts with biological systems (the definition of IUPAC). It can be classified into two big different categories based on the origin of the materials: firstly, the biological materials of natural origin; and secondly the synthetic ones formatted in a laboratory by different chemical methods. They are often used for medical purposes, so they perform, substitute or increase the function of a living system in a medical and biological way. Some of these applications are largely used today, for example, the artificial heart valve, the hip joint implant or the hernia mesh. This way the biomaterials are part of the day to day medicine like the surgery, odontology, cardiology and the transport of medicaments, which gives the possibility to the long way dosage of a medical product by an implant from the organism [4].

One of the greatest challenges to the treatment of a hernia is the biocompatibility of the different types of meshes which can guarantee its optimal integration into the host organism. For one can achieve adequate results, there is a need of detailed study of the different types of mesh; and, which is more important, the tracking of the response reactions of the organism like the angiogenesis, inflammation and the formation of the fibrous tissue $[5,6]$.

After the insertion of any kind of prosthesis, the host organism is subject to several processions during its recovery and the formation of the connective tissue. These proceedings are determined by the structure of the material, the properties of the filaments, the scale and the dimensions of the pores. The phases of the healing principles are similar to the new and bushy tissue which is on the way of form on the surface of the mesh which brings the characters. There are three values which are worth to follow from the point of histological view related to the interaction between the organism and the used substance: the degree of the tissue reaction; the density of the cells; and the fibroblast activity. During the short post operation period, which is considered between the first two weeks, the permeable macro-porous substances are more resistant than the not permeable ones [7-10].

The surgical hernia mesh implants used nowadays for the reconstruction of the abdominal wall are gaining more and more space into the medical field of study. This fact makes even more complex this segment of the surgery. The diverseness of the used meshes is increasing exponentially. This way, the manufacturers, being in competition, are trying to achieve the positive appreciation which associates the preferences for their products. These meshes are manufactured from diverse synthetic substances which are not breaking down and are biologically tolerated by the organism, respectively, they could be manipulated easily. Each of mesh types has its advantage and disadvantage, as well, like the adeptness theyintegrate, the degree of the formation of the connective tissue, anatomical preferences.

\section{Aims of Study}

We conducted an experimental study to compare the biointegration and tissue response of surgical meshes used nowadays, inserted in various layers of the abdominal wall.

*email: orsolyabauer@gmail.com; Phone: (+40)740-68 8606 
This investigation was made on animals and aimed to examine the distinct postoperative tissue reactions. From the aspect of the surgeon, the ideal mesh implant must have a minimal impact on generating of adhesions; its integration on the tissue level should be perfect; it must not crease; it must not shrivel; it should not generate infection; the possibility of seroma formation should be low; and to remain as less postoperative pain as possible. It is not negligible the fact that, beside all the abovementioned facts, the ideal mesh must be easy to fold, to cut and to manipulate and to be financially affordable [11].

During the animal experiments, we attended the comportment of two distinct types of abdominal wall meshes. The tests consisted of four main moments started from the preparations for the intervention and ending with the final processing of the data:

- anesthesia and surgical mesh insertion;

- histological sampling after 30 days of latency;

- Histopathological staining and examination;

- the procession and evaluation of results;

The final aim of the study was to find the first base for a leap forward to the everyday clinical practice using the outgoings of the experiment.

\section{Experimental part}

Material and methods

Study design

We completed an experimental study at the Experimental Station of the University of Medicine and Pharmacy of Tîrgu Mureo - Romania. We used two different types of mesh for the animal tests we made: Hernipro ${ }^{\circledR}$ / Biosintex with monofilament made of polypropylene; and mesh SM3 $\otimes / T H T$ Bio-science with multifilament made of Polyethylene-Terephthalate 3D (resin of the polyester family). They were placed on surgical way into the different layers of the abdominal wall and then they were fixed there. The summarized attributions of the above mentioned two meshes could be found in table 1.

\section{Experimental animals}

During our experiments, we used 30 female Wistar Albino rats which were kept in special prescribed cages at the Experimental Station of The University of Medicine and Pharmacy of Tirgu Mures. The rats were fed according to the appropriate standards in both pre- and post- operative period [12]. Their complex food consisted of wheat, corn, oat, wheat-flour, nutrition soy, sunflower seed, rape, bran and alfalfa. The daily necessity of food for the rats was depending on their ages and physiologic state. The daily need for a rat weighing $50 \mathrm{~g}$ was about $100 \mathrm{~g}$ of food. In case it was overw eighed one should consider $50 \mathrm{~g}$ of food for every $100 \mathrm{~g}$ of their extra weight.

\section{Preoperative Preparation}

Knowledge of the body mass of the rats was very important for surgical anesthesia, therefore in the preoperative period, each animal underwent on weight measurement and individual labeling. The average body mass in our case was 261 grams. They did not get any medication before the operation.

\section{Drugs applied during the anesthesia}

The importance and the role of the used anesthesia are to produce a loss of consciousness and myorelaxation which is painless during the surgical intervention, creating this way the optimal circumstances. A general anesthesia might be inducted using Ketamine $10 \%$ and Acepromazin Maleat (Sedam) of 10 milligrams accommodated to the body mass (milligrams $/ \mathrm{kg}$ body weight). The Sedam is responsible for the myorelaxation [13].

The anesthesia was set in 15-20 min and lasted for $5 \mathrm{~h}$. The determination of the exact narcotic was essential. Its over-dosage might lead to death, while the subdued dosage of anesthesia and myorelaxant might abridge the effectiveness of the surgical intervention and the life of the animal had been endangered as well. Surgical intervention: After the anesthesia was set and the myorelaxation was done, the animals were placed on the surgical table in dorsal position and the extremities were fixed. Rightbefore the surgery the superfluous hair was removed (epilation) and the disinfection of the zone was done. A midline of 22.5-centimeter-long incision was done for and circa $1.5 \mathrm{x}$ 1.5 centimeters defect was cut on the abdominal wall. In case where the subcutaneous implantation was performed we made skin and subcuticular tissue transection, if the mesh were inserted in sublay position (between the muscular fascia and the peritoneum) we sectioned the whole muscular layer of the abdominal wall. The two

\begin{tabular}{|c|c|}
\hline Polypropylene & PET 3D \\
\hline Monofilament & Multifilament \\
\hline Knitted material in rectangular shape & Knitted hexagonal("honeycomb") structure \\
\hline Pore size $1.3 \times 1.0$ & Pore size1.25x1.25 \\
\hline Thickness $0.59 \mathrm{~mm}$ & Thickness $0.65 \mathrm{~mm}$ \\
\hline Transparent resistant & Atraumatic softness particularly at the edges \\
\hline Biologically inert & Elasticity and excellent multidirectional mechanical \\
properties close to human tissue
\end{tabular}

Table 1

THE SUMMARIZED ATTRIBUTIONS OF THE MESHES USED IN THE STUDY

\begin{tabular}{|l|c|c|}
\hline PET 3D & Polypropylene & Control group \\
\hline 5 Onlay & 5 Onlay & 5 subcutan incision \\
\hline 5 Underlay & 5 Underlay & 5 muscular incison \\
\hline
\end{tabular}

Table 2

THE INSERTION PLACE OF THE TWO DIFFERENT MESHES IN THE LAYERS OF THE ABDOMINAL WALL 


\begin{tabular}{|l|l|}
\hline Measurement of neovascularization and fibrosis & Measurement of tissue inflammation \\
\hline$p=$ absence of fibrosis & $0=$ absence of inflammatory reaction \\
\hline $1=$ minimal fibrosis, fibroblast presence & $1=$ minimal lympho-plasmocytic infiltration \\
\hline $2=$ medium fibrosis & 2 = medium grade if inflammatory reaction \\
\hline$\beta=$ significant scar tissue & $\begin{array}{l}3=\text { significant inflammatory reaction, giant cell } \\
\text { presence }\end{array}$ \\
\hline
\end{tabular}

Table 3

MEASUREMENT OF THE INFLAMATORY REACTION AND FIBROSIS BY SEMI QUANTITATIVE MORPHOMETRY METHODS

Fig. la inserted directly on the peritoneum, the upper side being in contact with the muscular fascia. The last 10 rats were used as a control group as we made only incisions - 5 subcutaneous and 5 muscular longitudinal transections without using any prosthesis.

\section{Postoperative Period}

Right after the accomplishment of the surgical intervention a normal saline solution was administered to every animal in the form of subcutaneous injection. In the postoperative term the animals were fed with the habitual food associated with an adequate amount of fluids. We did not give any antibiotics. Tissue sampling was done after 30 days of latency. The euthanasia was made with ether. The abdominal wall was resected en-bloc.

Surgically removed bioptic samples were fixed in neutral buffered $4 \%$ formaldehyde solution and subsequently processed according to standard laboratory protocol (embedded in paraffin, 3-5 micron thick sections were made, xylene-ethanol sequence deparaffinization). Microscopic sections were stained using standard hematoxyline-eosin (HE), van Gieson (VG) and Masson's trichrome (M) protocols. HE stain was used to assess general histological architecture and cellular components, including the grade of inflammatory background. Special stains (VG, M) were used to evaluate the degree of fibrosis and overall tissue response to implants.

All microscopic slides were digitized using a Zeiss MiraxScan digital slide acquisition system [14].

\section{Results and discrussions}

From the 30 animals, subjects that underwent the procedure 2 perished during the postoperative period which might be explained by the intolerance of anesthesia. At the same time, there was one case where we observed postoperative complication, but it not imperiled the life of the animal. The complication consisted in granuloma formation on both, the macroscopic and microscopic histopathological sample.

The histological samples were examined by light microscopy. For the measurement of the inflammatory reaction and fibrosis quantity, semi quantitative morphometry methods were used ranging on a scale from 0 to 3 (table 3).

Figure $1 a($ PET 3D) and figure $1 b$ (Polypropylene) show that in either case the tissue reaction is present, however in case of PET 3D mesh the integration is better and a low rate of inflammatory reaction is observed (fig. 1a,b).
Fig. 2a

Fig. 2b 
Caused by the rigidity and the pore size, the Polypropylene figure $3 \mathrm{a}$ and figure $3 \mathrm{~b}$ mesh induces pronounced inflammatory reaction and in the process of fibrosis the presence of fibroblasts and granulation tissue is dominant. The mesh integration happens later than in case of PET implant, due to the presence of less coherent structures (fig. 3a,b).

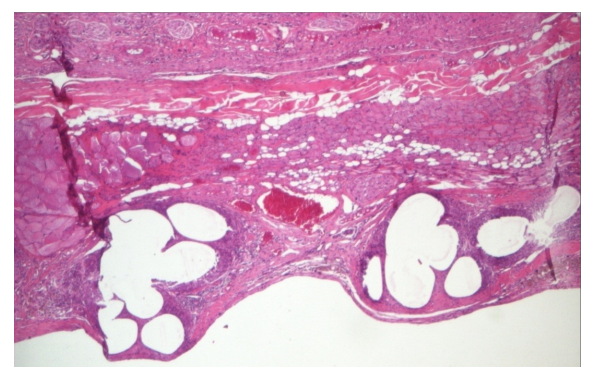

Fig. 3a

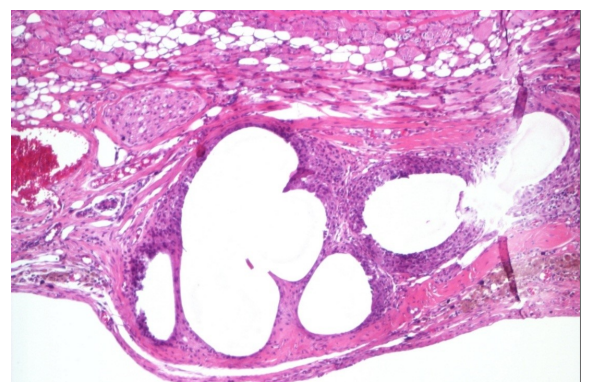

Fig. 3b

The comparison between PET 3D and Polypropylene result that mesh there is no significant difference in total individuals in both techniques regarding the rate of fibrosis (Fisher test, $p=0.1534$ ), but for 3D vs. Polypropylene mesh in underlay position, the statistical analysis shows a significant difference (Fisher test, $p=0.0476$ ) in favor of PET 3D mesh. This can be attributed to the difference of fibrosis which can be seen only if both meshes are in underlay position and adhere to both of the layers (fascia and peritoneum). The grade of the integration into subcuticular adipose tissue was lower.

In case of PET 3D mesh and control group (without any implant) there is a significant difference which suggests that in the case of PET 3D mesh there was a higher level of fibrosis (Fisher test $p=0.0007$ ). The Polypropylene mesh is compared to the control group and can be observed difference due to the properties of the mesh (material, pore size-width) the degree of fibrosis is reduced (Fisher test $p=0.0234)$. This is considered to the fact that the inflammation is represented more expressively which leads to a delayed fibrosis. Overall, the 3D PET mesh shows a greater significance compared to the control group.

As an overview, we can say that the development of the surgical meshes leads to an important development of the abdominal wall reconstruction. These days, further studies appear related to the experimental application of different materials for this reason, as well as, for their application in medical practice. Meshes made of Polyester are mainly used in Europe. They dispose of certain tissue reaction for it can produce fast fibroblast reaction, assuring this way their adequate fixation. Their grained texture generates their adhesion to the peritoneum and to the fascia and avoids with this attribution the slipping of the prosthesis from its original position.

The essential difference between the 3D PET and the Polypropylene mesh and their integration into the host organism is shown in the table (table 4).

Making a study to this chart there raises a last question namely: Which is the biological material worthwhile to use for surgical intervention? Here are coming in to consideration more aspects. The first of the point of view of the patient: less postoperative pain, less inflammatory reaction and a fast tissue ingrowth, all of these giving the result of short hospitalization. The decreased tissue tolerance also might cause recurrences as the time passes. During the last time, there were performed many clinical studies related to the above-mentioned issues. There were examined different types of meshes and their reactions to the animal and human tissues [15].

Similar experiments were made on pigs using three types of meshes manufactured by Covidien Company. It was the comparison of materials made from Polypropylene and two different Polyester resins. The results contained an estimation according to which the meshes made from Polyester were better from the point of view of the tissue integration, and, at the same time, the disposition of fibrous encapsulation had been reduced [16].

There is a Brazilian researching group who is working on the better integration of the Polypropylene mesh. They are examining the biocompatibility of the covered mesh with connective tissue on Wistar rats. The covered mesh appears to be more effective since it does not give rise to such isometric adhesions like the Polypropylene mesh [17].

It was important to follow the adhesion of the collagen coated Polyester mesh on one hand and the Polypropylene mesh on the other hand, which were implanted into an intraperitoneal position having the point of view the formation of adherence in the intraperitoneal level. As a result, there could not be found any differences in the tensile strength of the one covered with collagen and the Polypropylene mesh. But, on the point of view of their surface, the Polypropylene mesh had a better adherence to it [18].

An article published in the Hernia journal supports the hypothesis as our research that the use of low-weight large pore meshes is advantageous for abdominal wall function. It also demonstrates that the side effects of mesh implantation, comprising paresthesia and restriction of abdominal wall mobility is significantly affected by the type of material implanted. Of course, these upper mentioned characteristics are more important in the clinical practice use of the implants [19].

\begin{tabular}{|l|l|}
\hline 3D PET & Polypropylene \\
\hline Mature connective tissue cellular elements & Fibroblastic infiltration \\
\hline Lower grade of inflammatory reaction & Intensive lympho-plasmocytic infiltration \\
\hline Neovascularization presence & \\
& Reduced neovascularization \\
\hline Better tissue tolerance? & \\
\hline
\end{tabular}

Table 4

THE ESSENTIAL DIFFERENCE BETWEEN THE 3D PET AND THE POLYPROPYLENE MESH AND THEIR INTEGRATION 
If we are talking about the new aspects of the hernia surgery, we should mention a new approach to the inguinal hernia repair, the biologic implant. Compared with the synthetic ones they are characterized with reduced tissue integration and significant shrinkage, in case of polypropylene mesh the reticular collagen remodeling led to an augmentation of the scar due to significantly higher burst force resistance in comparison to native tissue [20].

In point of view of the patientrecovery and postoperative pain and not at least the operative time, the results of a cohort study (540 patients) describes better tolerance of Parietex ProGrip (polyester) mesh than the Polypropylene [21].

Another clinical retrospective study shows that the contact of tissues with the polypropylene mesh produces a local inflammatory response but the evolution towards surgical complications is limited and the advantage of the polypropylene is the favorable quality to price ratio [22].

\section{Conclusions}

The extended use of synthetic meshes in the hernioplasty is attributed to the necessity of polymer biological materials which ensure adequate consolidation to the abdomen and in vivo circumstance they dispose of qualities similar to the human tissue.

Having the results of our experiments, we can conclude:

- we did not observe any tissue intolerance, but differences were found in the degree of integration and tissue reaction, for example the PET 3D mesh integration happened sooner;

- the inflammation was almost equally present in both mesh types; however, the rate of fibrosis is greater in the case of PET 3D mesh;

- both types of meshes presented higher grade of adhesion to the muscular fascia and peritoneum layer;

- our experimental study confirms that fact, that does not exist a unique surgical procedure or implant which works properly in all cases, however, we consider that the in vivo experimental study on animals provides useful information to decide the optimal solution;

- the found results do not justify one or other implants supremacy, so in each case is important to point out that the surgeon must individualize his or her approach based on clinically experiences to perform the abdominal wall reconstruction. It is likely no single technique or prosthetic will accomplish the goals for all repairs.

\section{References}

1. ROSEN M. J., Atlas of Abdominal Wall Reconstruction, Philadelphia: Elsevier Saunders, 2012.

2. C., Sebeszet 7th ed., Budapest: Medicina, 2010.

3. ZOLLINGER R. M., ELLISON C., Zollinger's Atlas Of Surgical Operations, Mc Graw Hill Medical, 2011.

4. *** INTERNATIONAL UNION OF PURE AND APPLIED CHEMISTRY (IUPAC), Research Triangle Park, NC 27709-3757, USA, [Online]. Available: www.iupac.com.

5. RATNER D.B, HOFFMAN S. A, Biomaterials Science, Academic Press, 1996.
6. JUNGEK., BINNEBOSEL M., VON TROTHA K., ROSCH R., KLINGE U., NEUMANN U., LYNEN J ANSEN P., Mesh biocompatibility: effects of cellular inflammation and tissue remodelling, Langenbecks Arch Surg, vol. 397, no. 2, pp. 255-70, 2012 Feb.

7. ZOGBI, L., The Use Of Biomaterials To Treat Abdominal Hernias, [Online]. Available: http://www.intechopen.com/books/biomaterialsapplications-for-nanomedicine/the-use-of-biomaterials-totreatabdominal

8. VRIJ LAND W., Mesh Repair of Hernias of the abdominal wall, Rotterdam: Educost Publishers, 2003.

9. J., KUMAR V. AND ABBAS A., Robbins Basic Pathology, Philadelphia: Saunders Elsevier, 2007.

10. L. AND PORTELLA A., Retraction and fibroplasia in a polypropylene prosthesis: experimental study in rats,Hernia, vol. 14, no. 3, p. 291$298,2010$.

11.BELLOWSC., ALDER A.,HELTON W., Abdominal wall reconstruction using biological tissue grafts: present status and future opportunities, Expert Rev Med Devices, vol. 3, no. 5, pp. 657-75, Sep 2006.

12. MARINESCU B., COMAN C., Etica in experimentarea pe animale, Revista Româna de Bioetica, vol. 8, no. 3, pp. 96-103, Iulie - Septembrie 2010.

13. R., BROWN M. J., DANNEMAN P., ET AL, Anesthesia and Analgesia in Laboratory Animals, London: Elsevier, 2008.

14. MARCIAL GARCÍA R., GLORIA BUENO G., CARLOS P. M., Critical Comparison of 31 Commercially Available Digital Slide Systems in Pathology, International J ournal of Pathology, vol. 14, no. 4, pp. 285305, october 2006.

15. DONALD P. AND CHARLES B. E., Bioprosthetic Mesh in Abdominal Wall Reconstruction, vol. 26, no. 1, p. 18-24., 2012.

16. RAMSHAW R. G.-B., Comparison of Tissue Integration between Polyester and Polypropylene Prostheses in the Preperitoneal Space, The American Surgeon, vol. 69, no. 6, pp. 471-476, 2003.

17. RICCIARDI B. F., LUIZ H., ET AL, Abdominal hernia repair with mesh surrounded by fibrous tissue -experimental study in Wistar rats, Rev Col Bras Cir, vol. 39, no. 3, pp. 195-200, 2012.

18. BARBARA L., JOAO- VICENTE M., GROSSI B., BORGES M., ET AL, May polyester with collagen coating mesh decrease the rate of intraperitoneal adhesions in incisional hernia repair?, Arq Bras Cir Dig, vol. 26, no. 1, pp. 13-17, 2013.

19.WELTY G., KLINGE U., KLOSTERHALFEN B., ET AL, Functional impairment and complaints following incisional hernia repair with different polypropylene meshes, Hernia, vol. 5, no. 3, pp. pp 142-147, 2001.

20 GRUBER-BLUM S., BRAND J., KEIBL C., FORTELNY R., REDL H., MAYER F., PETTER-PUCHNER A., Abdominal wall reinforcement: biologic vs. degradable synthetic devices, Hernia, vol. 21, no. 2, pp. 305-315, 2017 Apr.

21. BATABYAL P., HADDAD R., WICKINS S. J. S., SWEENEY E., HUGH T., Inguinal hernia repair with Parietex ProGrip mesh causes minimal discomfort and allows early return to normal activities, Am J Surg, vol. 211, no. 1, pp. 24-30, 2016 Jan.

22. BRATU, D., BOICEAN A., TANASESCU C.SOFARIU, C., MIHETIU, A., CERNUSCA MITARIU I.S., OCNEAN, L, MOLDOVAN, C., BOITOR, C., Textile Polypropylene Allografts and their Postoperative Tissue Reaction in the Surgery of Inguinal Hernia, Mat Plast. 54, no. 1, 2017, p. 119

Manuscript received: 31.01 .2018 